\title{
AVR in patients with anomalous course of the circumflex artery without prosthetic downsizing
}

\author{
Luca Botta $^{1}$, Ciro Amodio ${ }^{1}$, Vincenzo Pagano ${ }^{1}$, Luca Di Marco ${ }^{2}$, Alessandro Leone ${ }^{1}$, \\ Antonino Loforte ${ }^{1}$, Sofia Martin-Suarez ${ }^{1}$, Carlo Savini ${ }^{1}$, and Davide Pacini ${ }^{3}$ \\ ${ }^{1}$ S. Orsola Hospital \\ ${ }^{2}$ s'Orsola Malpighi Hospital \\ ${ }^{3}$ University of Bologna
}

July 7, 2020

\begin{abstract}
An anomalous origin of the left circumflex coronary artery that arises as a side branch of the right coronary artery and encircles the aortic annulus is usually an incidental finding. However, in patients undergoing aortic valve/root procedures, its existence can significantly complicate the surgical treatment. We report our operative strategy with three different prostheses without valve downsizing.
\end{abstract}

\section{Surgical Technique}

Pre-operative evaluation for suspected coronary artery disease (CAD) with invasive coronary angiography should be performed when surgery or an intervention is planned to determine if concomitant coronary revascularization is indicated [1]. Coronary angiography is recommended before valve surgery in men $>40$ years and post-menopausal women, in patients with history of cardiovascular disease, symptoms of angina, objective evidence or suspected myocardial ischemia, decreased left ventricular systolic function and in case of cardiovascular/coronary risk factors [1]. Alternatively, coronary computed tomography (CT) can be used to rule out $\mathrm{CAD}$ in patients at low risk [2]. These imaging techniques can provide more information than the simple presence of CAD, such as coronary anatomy, origin, course and spatial relationships [1,2]. Nevertheless, coronary angiography and/or CT-scan are not always performed before aortic valve (AV) surgery (young patients without risk factors or urgent cases like endocarditis) or can be undervalued when stenotic lesions are excluded. Coronary anomalies such as anomalous left circumflex coronary artery (LCA) arising from the right sinus of Valsalva, strictly leaning on the aortic annulus, can have serious consequences in aortic valve/root surgery, particularly if unrecognized or underestimated [3,4]. In this paper, we present three patients with an anomalous course of the LCA undergoing AV replacement (AVR) with three different prostheses, without prosthetic downsizing.

Coronary angiography was performed in two patients. All patients underwent a preoperative CT-scan (Fig.1A). Chest was opened by median sternotomy and mild hypothermic cardiopulmonary bypass (32$34^{\circ} \mathrm{C}$ ) was initiated with ascending-aortic and right-atrial cannulation. After cross-clamping and infusion of antegrade crystalloid cardioplegia, aortotomy was performed with a transverse incision just above the sinotubular-junction avoiding the oblique incision towards the non-coronary sinus. Aortic valve was explored, leaving the native cusps in situ in presence of severe leaflet/annular calcifications to avoid any damage to the LCA during excision. A 1.0/1.5-mm coronary probe was placed into the assumed anomalous LCA to verify the anomalous course between the aortic root/annulus and the left atrial roof. A careful and complete dissection of the LCA was performed to free the artery from the aortic wall along its course, initially on the anterior 
and right side of the root, then posterior to the aorta at the level of the annulus until the atrio-ventricular groove (Fig.1B). Once the LCA was completely free from the surrounding tissues, we proceeded with native cusps excision. A meticulous attention was paid to the mobilized anomalous LCA when placing annular sutures (Fig.1C) using standard 2-0 U-stiches with small/soft pledgets on the ventricular side. Three different prostheses were used: a mechanical valve $(\mathrm{On}-\mathrm{X} 23 \mathrm{~mm})$ in a 31 years-old male with bicuspid aortic valve and severe regurgitation due to active endocarditis (E. Faecalis), a stented bioprosthesis (Carpentier Edwards magna Ease 23mm) and a rapid deployment sutureless valve (Perceval-S Size-M) in two ladies of 67 and 79 years with severe aortic stenosis, respectively. A complete LCA scheletonization allowed us to avoid prosthetic downsize, implanting valves of sizes exactly corresponding to every single annulus. Once the prosthesis was deployed, we verified the absence of compression on the LCA by the sewing-ring for stented prostheses (Fig.1D) or by the inflow-ring/sinusoidal struts for the sutureless valve. Aortotomy was closed in the standard fashion, avoiding the use of pledgets on the right side. After deairing and clamp removal, careful attention must be paid to LCA verifying its mobility with a blood-full root and the absence of hematoma, minor bleeding or compression by the surrounding structures. No sealants/hemostatic products were used. The patients were weaned from cardiopulmonary bypass in sinus rhythm without ischemic signs. Postoperative course was good in all cases without signs/symptoms of perioperative ischemia. Postoperative CT-scan confirmed the absence of LCA compression (Fig.2) in all patients, currently in good clinical conditions at last FU.

Anomalous origin of the coronary arteries is usually an incidental finding in patients undergoing cardiac catheterization. Coronary anomalies were found in 99 patients among 3,233 coronary angiograms by Sidhu and coll. [5], with an incidence of 3.06\%. Patients' mean age was 56.2years (range: 20-86). Ectopic origin of LCA from the right sinus or right coronary artery was noted in 13 patients $(0.40 \%)$, usually with a benign clinical course [5]. Appropriate imaging is crucial to understand the spatial relationship between the anomalous coronary arteries and the aortic valve/root before surgery but in the real life is not always performed, particularly in younger patients or in absence of coronary risk factors. In addition, the low incidence of this anomaly in clinical practice could reduce the proper attention of surgeons, particularly when critical stenoses are not reported. Anomalous LCA with a retro-aortic trajectory has major implications during AV surgery, particularly when unrecognized or underestimated [3-5]. Injury to the LCA may occur in isolated AVR by compression from the prosthesis (sewing ring) or ligation by annular sutures while resection of the non-coronary sinus or suturing could complicate aortic root/sinus repair. A careful surgical planning and management is mandatory and every step requires a strong attention as well as the choice of the right prosthesis. Implanting a smaller-sized prosthesis with the aim to avoid coronary artery distortion or compression has been reported [3,6], although patient-prosthesis-mismatch can occur with serious complications in small aortic annuli. In this small series, we have avoided this problem through the complete LCA skeletonization. When preoperative echocardiography shows a small aortic annulus and a standard stented prosthesis is not suitable, we suggest to use a sutureless aortic valve as reported by Cerillo and coll. [7]. In alternative, transcatheter aortic valve implantation has been described with success, using when necessary, a coronary guidewire before deploying TAVI to prevent coronary occlusion/stenosis [8]. Whenever postoperative ischemia should be detected after AVR with anomalous LCA, compression or surgical ligation must be immediately suspected and ruled out. If there is high suspicion of compression as the mechanism of ischemia, percutaneous coronary intervention can be a reasonable treatment option [6]. Coronary anatomy should always be evaluated in elective or urgent AVR. Careful and complete arterial skeletonization is recommended in presence of anomalous LCA to avoid prosthetic downsize and patient-prosthetic mismatch with every type of prosthesis.

\section{Figure Legend}

Fig.1 The yellow-arrow shows the anomalous LCA, originating from the right coronary artery (white-arrow) at multiplanar CT-scan (Fig.1A; Blu: aortic root; Red: coronary arteries). The LCA (black-arrow) was completely skeletonized and surrounded by a yellow vessel-loop before cusp removal (Fig.1B). U-stiches with soft-pledgets on the ventricular side were used, paying attention to avoid any LCA involvement (Fig.1C). After valve replacement, accurate evaluation of the field to verify the absence of LCA (black-arrow) compression (Fig.1D). 
Fig.2 Postoperative CT-scan after a mechanical valve replacement (white-arrow, Fig.2A) and after a sutureless implantation (red-arrow, Fig.2B). The yellow arrow shows the anomalous LCA, not compressed by the sewing/basal rings nor by the left atrium (L.A.).

\section{References}

1. Baumgartner H, Falk V, Bax JJ, et al. 2017 ESC/EACTS Guidelines for the management of valvular heart disease. Eur Heart J. 2017;38:2739-2791.

2. Opolski MP, Staruch AD, Jakubczyk M, et al. CT angiography for the detection of coronary artery stenoses in patients referred for cardiac valve surgery: systematic review and meta- analysis. J Am Coll Cardiol Img 2016;9:1059-70.

3. Liebrich M, Tzanavaros I, Scheid M, et al. Aortic valve/root procedures in patients with an anomalous left circumflex coronary artery and a bicuspid aortic valve: anatomical and technical implications. Interact Cardiovasc Thorac Surg. 2015;21:114-6.

4. Harky A, Hof A, Ahmad MU, et al. Incidental finding of anomalous circumflex coronary artery from right coronary sinus prior to aortic valve surgery. BMJ Case Rep. 2017Mar 31;2017.

5. Sidhu NS, Wander GS, MongaA, et al. Incidence, Characteristics and Atherosclerotic Involvement of Coronary Artery Anomalies in Adult Population Undergoing Catheter Coronary Angiography. Cardiol Res. 2019;10(6):358-368.

6. Castillo JG, Sanz J, Fischer GW, et al. Management of anomalous left circumflex artery encircling the aortic annulus in a patient undergoing multivalvular surgery. J Card Surg 2009;24:667-9.

7. Cerillo GA, Haxhiademi D, Berti S, et al. Sutureless Aortic Valve Replacement: An Easy and Safe Approach for Patients with Anomalous Left Circumflex Coronary Artery. J Heart Valve Dis. 2016;25:145148.

8. Ujihira K, Raval AN, Wolff MR, et al. Transcatheter aortic valve replacement in patients with anomalous left circumflex coronary artery. J Card Surg. 2019;34:503-505. 

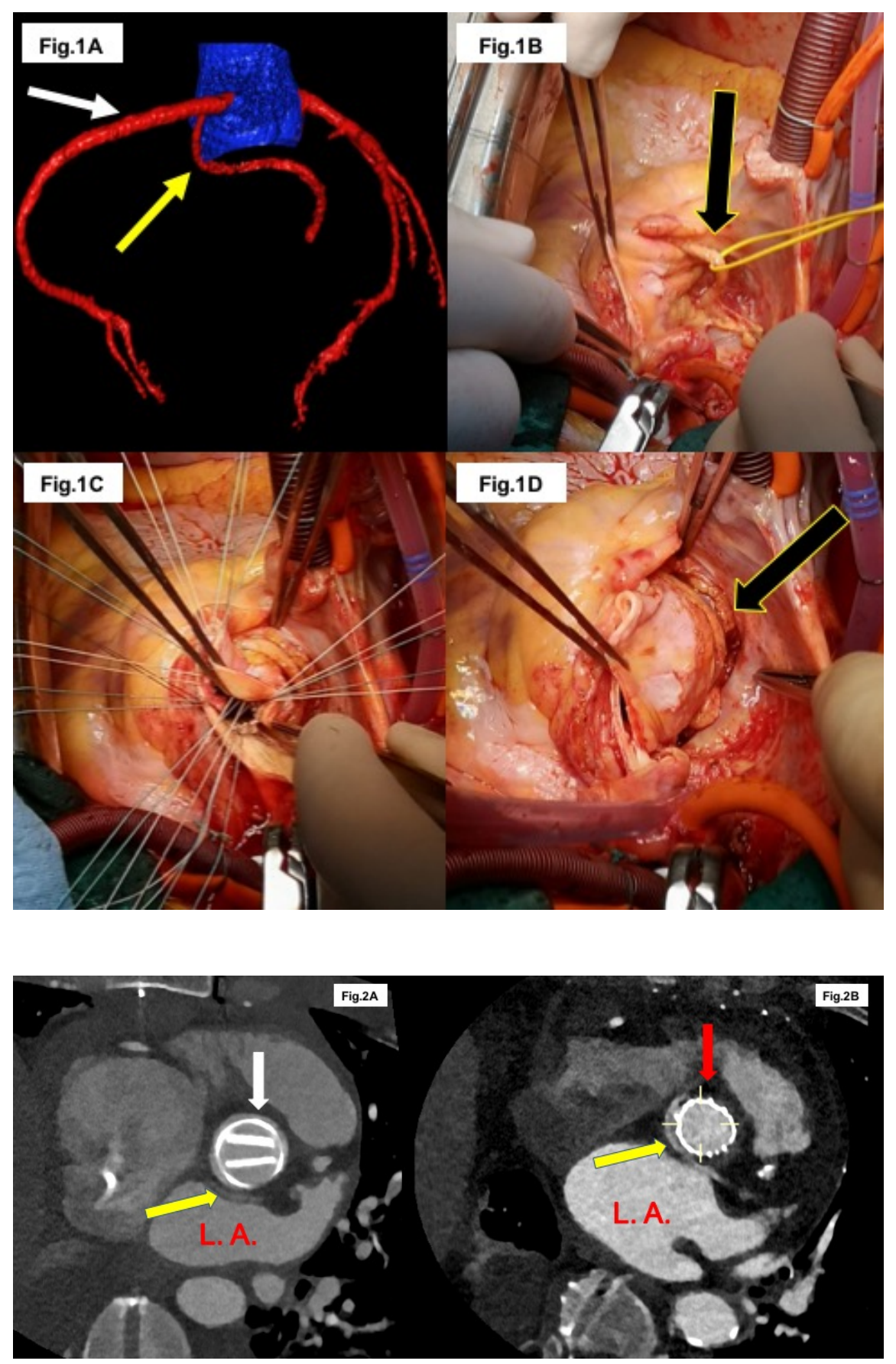